\title{
Evidence of Autophosphorylation in Txk: Y91 Is an Autophosphorylation Site
}

\author{
Jun-ichi Kashiwakura, ${ }^{a}$ Noboru Suzuki, ${ }^{b}$ Mitsuhiro Takeno, ${ }^{b}$ Saotomo Itoh, ${ }^{c}$ Teruaki OKu, ${ }^{a}$ \\ Tsuyoshi Sakane, ${ }^{b}$ Shizuo NakajIn, ${ }^{a}$ and Satoshi Toyoshima ${ }^{*, d}$ \\ ${ }^{a}$ Department of Biochemistry, Hoshi University; Shinagawa-ku, Tokyo 142-8501, Japan: ${ }^{b}$ Departments of Immunology \\ and Medicine, St. Marianna University School of Medicine; Kawasaki, Kanagawa 261-8511, Japan: ${ }^{c}$ Research Institute of \\ Tuberculosis, Japan Anti-Tuberculosis Association; Kiyose, Tokyo 204-8533, Japan: and ${ }^{d}$ Pharmaceuticals and Medical \\ Devices Evaluation Center, National Institute of Health Sciences; Minato-ku, Tokyo 105-8409, Japan.
}

Received November 29, 2001; accepted February 25, 2002

\begin{abstract}
We have previously shown that Txk, a member of Tec family tyrosine kinase, is expressed in Th1 and Th0 cells and directly contributes to gene transcription of Th1-related proteins, including interferon (IFN)- $\gamma$, through nuclear translocation in response to mitogenic stimuli. Btk, another member of Tec family tyrosine kinase, has been shown to have a Src family tyrosine kinase-dependent transphosphorylation site and an autophosphorylation site. However, little is known about the phosphorylation mechanism of Txk, except that 420 tyrosine residue was identified as the transphosphorylation site. In this study, we found that Txk autophosphorylated itself by using an in vitro kinase assay. To elucidate the role of phosphorylation in Txk function, we studied IFN- $\gamma$ secretion by Jurkat $T$ cells expressing mutant Txk proteins. While transfection with the wild-type Txk resulted in increased IFN- $\gamma$ production, the function was abrogated by disruption of the ATP biding site, which is presumably involved in the autophosphorylation mechanism. The results suggest that phosphorylated Txk is an active form to promote IFN- $\gamma$ synthesis. The 91 tyrosine residue of Txk is deduced to be an autophosphorylation site by comparing its structure with Btk. In Jurkat cells transfected with Txk Y91A, IFN- $\gamma$ production was decreased in comparison with the wild-type Txk transfected Jurkat cells. These data suggest that phosphorylation of the 91 tyrosine residue in Txk plays a positive regulatory role in Txk function.
\end{abstract}

Key words Txk; interferon- $\gamma$; autophosphorylation; ATP binding site

Tec family tyrosine kinases include Btk, Tec, Itk/Tsk/Emt, Bmx, and Rlk/Txk, and have been implicated in the regulation of immune function and hematopoiesis. ${ }^{1-10)}$ Tec, Itk and Txk play important roles in the differentiation and function of T cells. ${ }^{11-15)}$ Indeed, overexpression of Tec in T cells leads to enhanced gene expression of interleukin (IL)-2 mediated by TCR/CD3 or CD28 stimulation, while the introduction of a kinase dead Tec into T cells suppresses IL-2 gene expression. ${ }^{11,16)}$ Mice deficient in Itk display moderate impairment of both $\mathrm{T}$ cell development and peripheral $\mathrm{T}$ cell activation, whereas those deficient in Rlk/Txk show a slight decrease in the number of mature $\mathrm{T}$ cells, in particular of the $\mathrm{CD} 4^{+}$subset. ${ }^{17-19)}$ By contrast, the combined loss of Itk and Txk causes major $\mathrm{T}$ cell impairment. ${ }^{19)}$ The data suggest that Itk and Txk have a compensatory role with each other in mouse $\mathrm{T}$ cells. However, Txk appears to play a more critical role in the differentiation and function of Th1 cells in humans. We have previously shown that Txk is specifically expressed in Th1 and Th0 cells, but not Th2 cells, and that it upregulates interferon (IFN)- $\gamma$ production through gene transcription in response to mitogenic stimuli. ${ }^{20)}$ Although Txk shares Src homology ( $\mathrm{SH}) 3, \mathrm{SH} 2$, and tyrosine kinase catalytic domains with other Tec kinases, it lacks a pleckstrin homology $(\mathrm{PH})$ domain and has a nuclear localization signal sequence, which is responsible for nuclear translocation following TCR mediated signaling. ${ }^{9)}$ Like other Tec family kinases, Y420 of Txk was transphosphorylated by Fyn, a member of the Src family, after the engagement of TCR according to Chamorro and coworkers. ${ }^{21)}$ On the other hand, the role of autophosphorylation in Txk remains undetermined.

In this study, we determined whether an autophosphorylation mechanism operates in Txk by employing an in vitro ki- nase assay. We show here that Txk is autophosphorylated and that the phosphorylation of Y91, but not Y420, contributes to upregulated IFN- $\gamma$ gene transcription.

\section{MATERIALS AND METHODS}

Cell Jurkat cells were cultured in RPMI 1640 (ICN Biomedicals, Inc., Aurora, OH, U.S.A.) supplemented with $10 \%$ fetal calf serum (Sanko Junyaku Co., Tokyo), penicillin and streptomycin.

Antibodies Purified and biotinylated anti-IFN- $\gamma$ monoclonal antibody (mAb), anti-IL-2 mAb, anti-IL-4 mAb, and PY20, anti-phosphotyrosine antibody were purchased from PharMingen (San Diego, CA, U.S.A.). Anti-human Txk Abs were purchased from Santa Cruz Biotechnology (Goat: Santa Cruz, CA, U.S.A.) and obtained from rabbits immunized with a peptide (CSVQKRQMRTQISLSTDEEL) as described by Kikuchi et al. ${ }^{22}$

Plasmids The vector pME18S-txk, containing fulllength wild-type human txk cDNA in a mammalian expression vector, pME18S (SR- $\alpha$ promoter), was generated as previously described. ${ }^{20)}$ The point mutant of Txk was created using the QuickChange site-directed mutagenesis kit (Stratagene, La Jolla, CA, U.S.A.). Briefly, pME18S-txk was used as a template. The amplification cycles consisted of 1 cycle of denaturation $\left(95^{\circ} \mathrm{C}\right)$ for $1 \mathrm{~min}$, followed by 16 cycles of denaturation $\left(95^{\circ} \mathrm{C}\right)$ for $30 \mathrm{~s}$, annealing for $1 \min \left(55^{\circ} \mathrm{C}\right)$, and polymerization for $10 \mathrm{~min}\left(68^{\circ} \mathrm{C}\right)$. The PCR product was treated with Dpn I, which is specific for methylated DNA, and the synthesized nonmethylated DNA containing the desired mutation was recovered. The resultant mutant vector was used for the transformation of E. coli JM109. We gener- 
ated pME18S-txk K299E, pME18S-txk K121E, pME18S-txk Y91A and pME18S-txk Y420A by using the following primers: K299E forward 5'-CATATCCAGGTAGCTATCGAGGCCATCAATGAAGGCTCC-3' and K299E reverse 5'-GGAGCCTTCATTGATGGCCTCGATAGCTACCTGGATATG-3'. K121E forward 5'-AATCCTCACTGGTGGGAGGCAAGAGACCGTTTG-3' and K121E reverse 5' -CAAACGGTCTCTTGCCTCCCACCAGTGAGGATT-3'. Y91A forward 5'-CCAAGTCAAGGCACTTGCTGATTTTCTGCCCAGAG-3' and Y91A reverse 5' -CTCTGGGCAGAAAATCAGCAAGTGCCTTGACTTGG-3'. Y420A forward 5'-GTTTTGGATGATGAGGCTGTCAGTTCTTTTGGAG-3' and Y420A reverse 5'-CTCCAAAAGAACTGACAGCCTCATCATCCAAAAC-3'. Fidelity of all the constructs was confirmed by DNA sequencing. To generate a prokaryotic expression vector, cDNAs encoding the wildtype and mutant Txk were inserted into pQE31 (QIAGEN, Valencia, CA, U.S.A.). The resultant plasmids were designated pQE-txk, pQE-txk Y91A, and pQE-txk Y420A.

Transfection and Stimulation of Jurkat Cells Purified plasmids were transfected into Jurkat cells by electroporation, as described. ${ }^{20)}$ After incubation for $48 \mathrm{~h}$, transfected Jurkat cells were stimulated with PHA (Sigma, St. Louis, MO, U.S.A.) for another $24 \mathrm{~h}$. In the preliminary experiment we used pRSV-CAT to determine transfection efficiency. We found that differences in the transfection efficiencies of each transfection were negligible in the experiments.

ELISA Amounts of IFN- $\gamma$, IL-2 and IL-4 in cultured supernatants of the transfected Jurkat cells were measured by ELISA, as described previously. ${ }^{23)}$ Detection was performed with an ECL detection kit (Amersham Pharmacia Biotech, Piscataway, NJ, U.S.A.).

Expression and Purification of Recombinant Human Txk and Mutant Proteins The expression vector, pQE31, containing the entire coding region of human txk cDNA was used for the transformation of E. coli JM109 cells. Expression of Txk proteins were induced by isopropyl $\beta$-D-thiogalactopyranoside (IPTG: Wako Pure Chemical Industries, Osaka) for $4 \mathrm{~h}$ at $37^{\circ} \mathrm{C}$. After cellular proteins were eluted from JM109 by sonication, the recombinant proteins were purified by immunoprecipitation using rabbit anti-human Txk antibody.

In Vitro Kinase Assay Recombinant Txk proteins were washed by a kinase buffer, including 40 mм HEPES (pH 7.4), $10 \mathrm{mM} \mathrm{MgCl}_{2}, 3 \mathrm{~mm} \mathrm{MnCl}$ and $1 \mathrm{~mm}$ DTT. After incubation in $40 \mu \mathrm{l}$ kinase buffer containing $250 \mu \mathrm{M}$ ATP at $25^{\circ} \mathrm{C}$ for $5 \mathrm{~min}$, the proteins were subjected to sodium dodecyl sulfatepolyacrylamide gel electrophoresis (SDS-PAGE), then transferred onto a PVDF membrane. On the membrane, Txk and phosphotyrosines were detected by western blot analysis using goat anti-human Txk and PY20 antibodies, respectively.

Statistical Analysis At least three independent experiments were performed for each assay. All data are expressed as the mean and S.D. The statistical significance of differences between the means was determined by applying a twotailed Student's $t$-test. A difference was considered statistically significant with $p<0.05$.

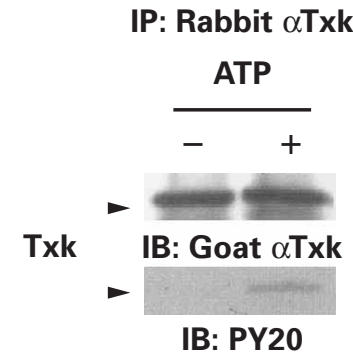

Fig. 1. Autophosphorylation of Txk

E. coli JM109 was transformed with pQE31 (mock) and pQE-txk (Txk). The cells were cultured with IPTG for $4 \mathrm{~h}$, recombinant Txk proteins were recovered by immunoprecipitation with rabbit anti-Txk. The recovered proteins were incubated in kinase buffer in the presence or absence of ATP. Thereafter, the proteins were immunoblotted with goat anti-Txk (top panel) and anti-phosphotyrosine (PY20) (bottom panel).

\section{RESULTS}

Autophosphorylation of Human Txk It has been shown that the function of Tec family tyrosine kinase, such as Btk, is regulated by autophosphorylation as well as transphosphorylation. ${ }^{24,25)}$ Txk is phosphorylated and activated by the Src family kinase, Fyn, in the signaling cascade triggered by $\mathrm{T}$ cell receptor engagement. ${ }^{21)}$ However, it remains undetermined whether Txk autophosphorylates itself. To analyze the autophosphorylation of Txk by in vitro kinase assay, we generated a prokaryotic Txk expression vector, pQE-txk, containing human txk cDNA. Recombinant human Txk protein was eluted by immunoprecipitation. After incubation of the recovered protein in kinase buffer with or without ATP in vitro, phosphotyrosine was detected by the immunoblotting technique using PY20. We found that Txk was phosphorylated in the presence of exogenous ATP, indicating that Txk has the potential to phosphorylate itself (Fig. 1).

Involvement of K299 at the ATP Binding Site in IFN- $\gamma$ Production by $\mathbf{T}$ Cells We have previously demonstrated that Txk translocates into nuclei in response to mitogenic stimuli and upregulates IFN- $\gamma$ gene transcription. ${ }^{20)}$ Like other members of Tec family tyrosine kinase, it is plausible that Txk function is regulated by transphosphorylation and autophosphorylation. It has been shown that the ATP biding site following the $\mathrm{SH} 2$ domain is involved in the phosphorylation process as a phosphate donor. ${ }^{26)}$ To clarify the role of kinase activity in Txk, we constructed Txk mutant expression vectors, pME18S-txk K299E, in which glutamic acid was substituted for lysine at the ATP biding site, and pME18Stxk K121E, in which a point mutation was introduced to a site irrelevant to ATP binding. We found that the kinase activity of Txk was lost in K299E, but maintained in K121E. We compared the effects of these Txk mutants on IFN- $\gamma$ production by Jurkat cells. In preliminary experiments using a cotransfection system of pRSV-CAT, we found that our procedure yielded almost stable transfection efficiency, irrespective of the plasmids. ${ }^{20)}$ Transfection of the wild-type Txk resulted in increased IFN- $\gamma$ synthesis. Transfection of K299E failed to enhance IFN- $\gamma$ synthesis (Fig. 2a). In contrast, Jurkat cells transfected with K121E produced IFN- $\gamma$ as much as those with the wild-type Txk (Fig. 2b). IL-2 production was not significantly affected by any Txk mutants studied here. The results suggest that kinase activity, in which the ATP binding site including 299 lysine residue is involved, is 

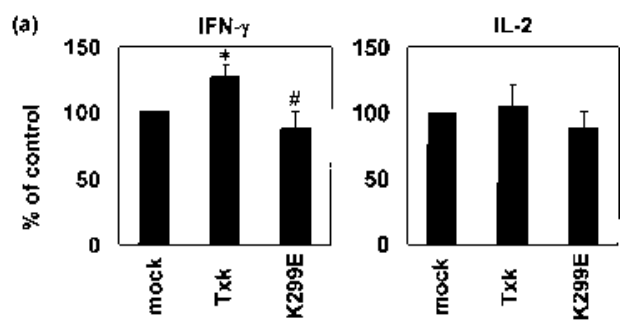

(b)

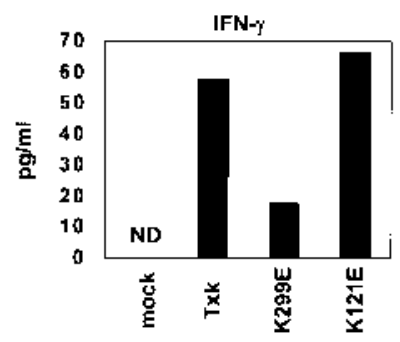

Fig. 2. Effects of Disrupted ATP Biding Site of Txk on IFN- $\gamma$ Production Jurkat cells were transfected with either pME18S (mock), pME18S-txk (Txk), pME18S-txk K299E (K299E), or pME18S-txk K121E (K121E), 48 h later, Jurkat cells were stimulated with PHA for another $24 \mathrm{~h}$. Concentrations of cytokines in the culture supernatants were measured by ELISA. (a) Mean \pm S.D. in standardized percentages of individual data to mock controls in each experiment are shown. IFN- $\gamma$ and IL-2 production by Jurkat cells transfected with the mock was $20.6 \pm 13.5 \mathrm{pg} / \mathrm{ml}$ and $2754.39 \pm 503.26 \mathrm{pg} / \mathrm{ml}$ (mean \pm S.D.), respectively, in three independent experiments. (b) Representative data of IFN- $\gamma$ production in Jurkat cells transfected with either the mock, wild-type Txk, K299E or K121E, are shown. ND denotes not detectable. ${ }^{*} p<0.05 v s$. mock transfected Jurkat cells. ${ }^{~} p<0.05 v s$. pME18S-txk transfected Jurkat cells.

necessary for enhancing IFN- $\gamma$ synthesis by activated Txk.

Autophosphorylation Sites of Txk and Production of IFN- $\gamma$ In Btk, 551 tyrosine residue has been identified as the transphosphorylation site by Src family kinases, whereas 233 tyrosine residue has been identified as the autophosphorylation site. ${ }^{24,25}$ Based on the homology between Btk and Txk, we deduced that the corresponding transphosphorylation site is 420 tyrosine residue, and that the autophosphorylation site is 91 tyrosine residue, respectively. Indeed, a recent study identified the 420 tyrosine residue of Txk as the transphosphorylation site by Fyn. ${ }^{21)}$ We generated pQE-txk Y91A and Y420A by substitution from tyrosine to alanine at Y91 and Y420 of Txk, respectively. We examined whether the mutant Txk proteins autophosphorylated itself by using an in vitro kinase assay. Expression levels of Txk and phosphorylated tyrosine were determined by signal intensities of immunoblotting with anti-Txk antibody and PY20, respectively. The relative activity of phosphorylation was calculated by dividing the phosphorylated protein level by the Txk expression level. We found that the relative activity was remarkably reduced in Y91A and Y420A compared with the wild-type Txk (Fig. 3). The results suggest that the tyrosine residues at both 91 and 420 are phosphorylated by the kinase activity of Txk itself.

To elucidate the relationship between the autophosphorylation sites of Txk and IFN- $\gamma$ synthesis, we measured the amounts of IFN- $\gamma$ produced by Jurkat cells transfected with pME18S-txk Y91A and Y420A. Transfection of the wildtype Txk resulted in increased IFN- $\gamma$ production. In contrast, transfection with Y91A secreted reduced amounts of IFN- $\gamma$ in comparison with the wild-type Txk. On the other hand, transfection with Y420A did not inhibit the secretion of IFN$\gamma$ compared with transfection of wild-type Txk (Fig. 4).

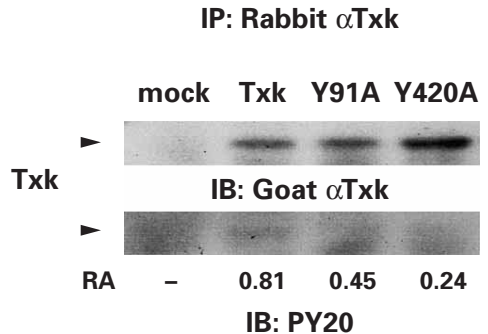

Fig. 3. Autophosphorylations of Y91A and Y420A

E. coli JM109 was transformed with pQE31 (mock), pQE-txk (Txk), pQE-txk Y91A (Y91A), and pQE-txk Y420A (Y420A). The cells were cultured with IPTG for $4 \mathrm{~h}$, then recombinant Txk and mutant proteins were recovered by immunoprecipitation with rabbit anti-Txk. The recovered proteins were incubated in kinase buffer in the presence or absence of ATP. Thereafter, the proteins were immunoblotted with goat anti-Txk (top panel) and anti-phosphotyrosine (PY20) (bottom panel). RA indicates relative activity, which is the ratio of signal intensity of anti-phosphotyrosine blotting to anti-Txk blotting. Substitutions at both Y91 and Y420 remarkably reduced the autophosphorylation level.

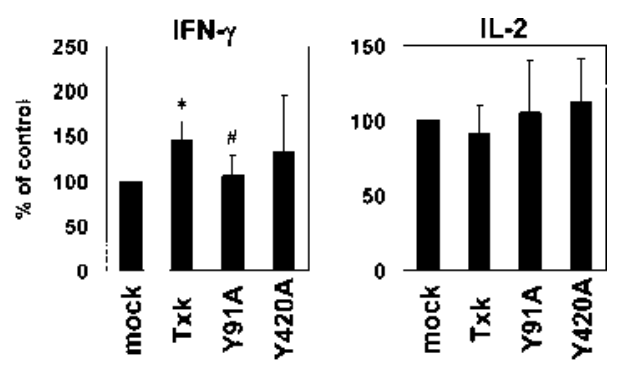

Fig. 4. Effects of Y91A and Y420A Transfection on IFN- $\gamma$ Production by Jurkat Cells

IFN- $\gamma$ production was measured by PHA-stimulated Jurkat cells which were transfected with either pME18S (mock), pME18S-txk (Txk), pME18S-txk Y91A (Y91A), or pME18S-txk Y420A (Y420A). The graph shows the mean \pm S.D. in standardized percentages of individual data to mock controls in each experiment. ${ }^{*} p<0.05 v s$. mock transfected Jurkat cells. ${ }^{\#} p<0.05$ vs. pME18S-txk transfected Jurkat cells.

None of the expression vectors studied here affected IL-2 production. These results suggest that phosphorylation at 91 tyrosine residue is critical for the activation of Txk, leading to the upregulation of IFN- $\gamma$ gene transcription.

\section{DISCUSSION}

In this study, we demonstrate that Txk is autophosphorylated by itself, and that the phosphorylation level of Txk is closely associated with the activation of Txk. Furthermore, we show that 91 tyrosine residue is an important phosphorylation site to positively regulate the function of Txk, in addition to 420 tyrosine residue, which was previously identified as a transphosphorylation site by a Src family kinase, Fyn. ${ }^{21)}$

It is becoming clear that Th1 and Th2-specific transcription factors play critical roles in Th1 and Th2 cell maturation, respectively. T-bet, a Th1-specific transcription factor, is shown to transactivate IFN- $\gamma$ gene expression, while GATA3 and c-Maf are known to be selectively involved in Th2 cell specific cytokine production. ${ }^{27-32)}$ We recently found that Txk functions as a Th1-specific transcription factor to promote Th1-related proteins, including IFN- $\gamma$, TNF- $\beta$, and CCR5 (unpublished observation). Therefore, the Txk-mediated signaling pathway is one possible pharmacological target involved in the regulation of Th1 and Th2 balance in immune responses.

Tec kinases contain $\mathrm{SH} 3, \mathrm{SH}$, and tyrosine kinase cat- 
alytic domains as Src family kinases. ${ }^{9)}$ The importance of the Tec kinases in antigen receptor signaling was first demonstrated by the observation that mutations of the btk gene are associated with a profound immunodeficiency, X-linked agammaglobulinemia (XLA). In patients with XLA, B cell development and immunoglobulin synthesis were defective because of impaired B cell receptor (BCR) signaling. ${ }^{33,34)}$ Cross-linking of BCR leads to the activation of Src family kinases, which in turn phosphorylate Btk. Phosphopeptide mapping and mutational analyses of Btk have identified 551 tyrosine as the transphosphorylation site by Src family kinases. ${ }^{24)}$ In addition, 233 tyrosine residue was identified as the autophosphorylation site. ${ }^{25)}$ Interestingly, the phosphorylated Btk, in which the 233 tyrosine residue of the SH3 domain is autophosphorylated, has been shown to display distinct ligand binding properties from the nonphosphorylated Btk. While the nonphosphorylated Btk binds to c-Cbl and Wiscott-Aldrich syndrome protein (WASP), the autophosphorylated Btk binds to c-Cbl, but not to WASP, and acquires a high affinity for Syk. ${ }^{35}$ ) These findings suggest that the autophosphorylation in Btk is implicated in the selection of ligands against Btk. While the autophosphorylation plays a negative regulatory role in the function of Src family kinases, activation of Btk requires Btk kinase activity, the putative autophosphorylation site (Y551), and most notably, a functional PH domain. ${ }^{24,36)}$

Like other Tec family members, Txk is transphosphorylated by the Src family in TCR signaling. The notion is supported by our previous findings that no IFN- $\gamma$ is secreted by Txk transfected Jurkat cells without stimulation. Recently, the critical transphosphorylated site has been identified at 420 tyrosine residue. ${ }^{21)}$ As deduced from the homology between Btk and Txk, we show here that 91 tyrosine residue is another phosphorylation site, which is possibly mediated by an autophosphorylation mechanism. Because the transfection of a substitution mutant, Y91A, to Jurkat cells failed to support an increased IFN- $\gamma$ synthesis in response to mitogenic stimuli, the autophosphorylation mechanism at the residue may play a critical role in Txk function as a Th1-specific transcription factor.

Acknowledgement We are grateful to H. Nagafuchi for helpful comments. We are also indebted to Mr. S. Tada, Ms. Y. Yamada, Ms. Y. Kashiwagi and Ms. N. Kato for their excellent technical assistance. This work was supported with funding from Hoshi University Otani Research Grants, 2001 Grant for the promotion of the advancement of education and research in graduate schools, 2001 grant-in-aid for scientific research project No. 13037033 from the Ministry of Education, Culture, Sports, and Technology of Japan, 2001 Science and Technology of the Japanese Government, Special Coordination Funds of the Ministry of Education, Culture, Sports, Science and Technology of the Japanese Government, and 2001 Comprehensive Research on Aging and Health of the Health Science Research Grants of the Ministry of Health, Labour, and Welfare in Japan.

\section{REFERENCES}

1) Bolen J. B., Curr. Opin. Immunol., 7, 306-311 (1995).

2) Rawlings D. J., Witte O. N., Semin. Immunol., 7, 237-246 (1995).
3) Mano H., Int. J. Hematol., 69, 6-12 (1999).

4) Smith C. I., Baskin B., Humire-Greiff P., Zhou J. N., Olsson P. G., Maniar H. S., Kjellen P., Lambris J. D., Christensson B., Hammarstrom L., J. Immunol., 152, 557-565 (1994).

5) Vetrie D., Vorechovsky I., Sideras P., Holland J., Davies A., Flinter F., Hammarstrom L., Kinnon C., Levinsky R., Bobrow M., Smih C. I., Bentley D., Nature (London), 361, 226-233 (1993).

6) Mano H., Ishikawa F., Nishida J., Hirai H., Takaku F., Oncogene, 5, 1781-1786 (1990).

7) Gibson S., Leung B., Squire J. A., Hill M., Arima N., Goss P., Hogg D., Mills G. B., Blood, 82, 1561-1572 (1993).

8) Tamagnone L., Lahtinen I., Mustonen T., Virtaneva K., Francis F., Muscatelli F., Oncogene, 9, 3683-3688 (1994).

9) Haire R. N., Ohta Y., Lewis J. E., Fu S. M., Kroisel P., Litman G. W., Hum. Mol. Genet., 3, 897-901 (1994).

10) Ohta Y., Haire R. N., Amemiya C. T., Litman R. T., Trager T., Riess O., Litman G. W., Oncogene, 12, 937-942 (1996).

11) Yang W. C., Ghiotto M., Barbarat B., Olive D., J. Biol. Chem., 274, 607-617 (1999).

12) Perez-Villar J. J., Kanner S. B., J. Immunol., 163, 6435-6441 (1999).

13) Ching K. A., Kawakami Y., Kawakami T., Tsoukas C. D., J. Immunol., 163, 6006-6013 (1999).

14) Sommers C. L., Rabin R. L., Grinberg A., Tsay H. C., Farber J., Love P. E., J. Exp. Med., 190, 1427-1438 (1999).

15) Schneider H., Schwartzberg P. L., Rudd C. E., Biochem. Biophys. Res. Commun., 252, 14-19 (1998).

16) Yang W. C., Olive D., Eur. J. Immunol., 29, $1842-1849$ (1999).

17) Schaeffer E. M., Broussard C., Debnath J., Anderson S., McVicar D. W., Schwartzberg P. L., J. Exp. Med., 192, 987-1000 (2000).

18) Liu K. Q., Bunnell S. C., Gurniak C. B., Berg L. J., J. Exp. Med., 187, $1721-1727$ (1998).

19) Schaeffer E. M., Debnath J., Yap G., McVicar D., Liao X. C., Littman D. R., Sher A., Varmus H. E., Lenardo M. J., Schwartzberg P. L., Science, 284, 638-641 (1999).

20) Kashiwakura J., Suzuki N., Nagafuchi H., Takeno M., Takeba Y., Shimoyama Y., Sakane T., J. Exp. Med., 190, 1147-1154 (1999).

21) Chamorro M., Czar M. J., Debnath J., Cheng G., Lenardo M. J., Varmus H. E., Schwartzberg P. L., BMC Immunol., 2, 3 (2001).

22) Kikuchi H., Imajoh-Ohmi S., Kanegasaki S., Biochim. Biophys. Acta, 1162, 171-176 (1993).

23) Kosuge T., Tamura T., Nariuchi H., Toyoshima S., Biol. Pharm. Bull., 3, 1-5 (2000).

24) Rawlings D. J., Scharenberg A. M., Park H., Wahl M. I., Lin S., Kato R. M., Fluckiger A. C., Witte O. N., Kinet J. P., Science, 271, 822825 (1996).

25) Park H., Wahl M. I., Afar D. E., Turck C. W., Rawlings D. J., Tam C., Scharenberg A. M., Kinet J. P., Witte O. N., Immunity, 4, 515-525 (1996).

26) Donnadieu E., Lang V., Bismuth G., Ellmeier W., Acuto O., Michel F., Trautmann A., J. Immunol., 166, 5540-5549 (2001).

27) Szabo S. J., Kim S. T., Costa G. L., Zhang X., Fathman C. G., Glimcher L. H., Cell, 100, 655-669 (2000).

28) Mullen A. C., High F. A., Hutchins A. S., Lee H. W., Villarino A. V., Livingston D. M., Kung A. L., Cereb N., Yao T. P., Yang S. Y., Reiner S. L., Science, 292, 1907-1910 (2001)

29) Ray A., Cohn L., J. Clin. Invest., 104, 985-993 (1999).

30) Nawijn M. C., Dingjan G. M., Ferreira R., Lambrecht B. N., Karis A., Grosveld F., Savelkoul H., Hendriks R. W., J. Immunol., 167, 724732 (2001).

31) Christodoulopoulos P., Cameron L., Nakamura Y., Lemiere C., Muro S., Dugas M., Boulet L. P., Laviolette M., Olivenstein R., Hamid Q., J. Allergy Clin. Immunol., 107, 586-591 (2001).

32) Li B., Tournier C., Davis R. J., Flavell R. A., EMBO J., 18, 420-432 (1999).

33) Smith C. I., Islam K. B., Vorechovsky I., Olerup O., Wallin E., Rabbani H., Baskin B., Hammarstrom L., Immunol. Rev., 138, 159-183 (1994).

34) Ellmeier W., Jung S., Sunshine M. J., Hatam F., Xu Y., Baltimore D., Mano H., Littman D. R., J. Exp. Med., 192, 1611-1624 (2000).

35) Morrogh L. M., Hinshelwood S., Costello P., Cory G. O., Kinnon C., Eur. J. Immunol., 29, 2269-2279 (1999).

36) Mahajan S., Fargnoli J., Burkhardt A. L., Kut S. A., Saouaf S. J., Bolen J. B., Mol. Cell. Biol., 15, 5304-5311 (1995). 